\title{
Erratum to: Experienced high performance work system, extroversion personality, and creativity performance
}

\author{
Yun-Hwa Chiang ${ }^{1}$ - Chu-Chun $\mathrm{Hsu}^{2} \cdot$ Hsi-An $\mathrm{Shih}^{3}$
}

Published online: 7 June 2015

(C) Springer Science+Business Media New York 2015

\section{Erratum to: Asia Pac J Manag (2015) 32:531-549 DOI 10.1007/s10490-014-9403-y}

The author group in the original version of this article contained a mistake. The last author's name should be Hsi-An Shih, instead of His-An Shih. The correct name appears above.

The online version of the original article can be found at http://dx.doi.org/10.1007/s10490-014-9403-y.

Chu-Chun Hsu

hsucc@mail.stust.edu.tw

Yun-Hwa Chiang

hui3789@yahoo.com.tw

Hsi-An Shih

hashih1224@gmail.com

1 Department of Business Administration, Ming Chuan University, 250, Sec. 5, Chung Shan N. Rd., Taipei 111 Taiwan, Republic of China

2 Department of International Business, Southern Taiwan University of Science and Technology, No. 1, Nan-Tai Street, Yongkang Dist., Tainan City 710 Taiwan, Republic of China

3 Department of Business Administration, National Cheng Kung University, 1 University Road, Tainan 701 Taiwan, Republic of China 